\title{
Effect Of Retention Sutures For Prevention Of Abdominal Wound Dehiscence After Laparotomy In High Risk Patients(A Prospective Study).
}

\author{
Mohanad abdulretha \\ .FI.C.M.S.) Department of surgery College of medicine Thi-qar University
}

\begin{abstract}
BACKGROUND: Wounds and their management are fundamental to the practice of surgery.Surgical wound dehiscence after laparotomy remains a serious complication.To evaluate the effect of prophylactic retention sutures in patients with a high risk for wound dehiscence who underwent midline laparotomy. Patients and methods:One hundred fifty(150) cases were randomized to form two groups with 75 patients each: a prophylactic group by using retention sutures and an non prophylactic group. A central randomization for both hospitals was performed. Two patients in the non prophylactic group and three patients in the prophylactic group In the non prophylactic(control) groupStandard midline incision and continuous mass closure tech-nique was used in each case using a running looped 1/0 nylon string located $1 \mathrm{~cm}$ from the edge of the lineaalba.In the prophylactic group, the fascia was sutured using the same technique as the non prophylactic group; however, retention sutures were added using a 1/0 nylon string every $10 \mathrm{~cm}$ and contained $5 \mathrm{~cm}$ of the skin, subcutaneous tissue, rectus muscle, and abdominal fascia (except peritoneum) on each side. All fascia closures were performed by tow attending surgeons who adhered strictly to the protocol.Occurrence of abdominal dehiscence was assesseddaily by precise examination of the wound. Results:The incidence of abdominal wound dehiscence was 3 patients (4\%) in the prophylactic group and 10 patients (13.3\%) in the control (nonprophylactic )group $(P=0.007)$ therefore it is significantConclusion:Ourconclsion that prophylactic retention sutures can decrease the incidence of abdominal wound dehiscence but although there is decrease incidence of post operative evisceration, wound infection and post operative pain, there was no significant difference.
\end{abstract}

\section{INTRODUCTION}

Surgical wound dehiscence after laparotomy remains a serious complication. It presents a mechanical failure of wound healing of surgical incisions. Surgical incisions stimulate the healing process which in reality is a complex and continous process with four different stages: Hemostasis, .inflammation, proliferation, and maturation [1] During hemostasis, platelets aggregate, degranulate and activate blood clotting. The clot is degrading, the capillaries dilates and fluids flow to the wound site, activating thecomplement cascade. Macrophages, lysis of cells and neutrophills are a source of cytokines and growth factors that are essential for normal . wound healing [1,2] Wounds and their management are fundamental to the practice of surgery. Any surgical intervention will result in a wound. The surgeon's task is to minimize the adverse effects of the wound, remove or repair damaged structures and harnessthe process of wound healing to restore function General surgeons make various abdominal incisions. Disruption of abdominal surgical wound is one of the common causes of early relapartomy[3]. Till recent, however, it has been a subject little understood with little known about its exact etiopathogenesis, there was little a surgeon could do to take preventive steps [4]. Abdominal wound dehiscence or burst abdomen is one of the most serious postoperative complications and is associatedwith high morbidity and mortality. It occurs with an incidence of $0.4 \% \mathrm{e} 3.5 \%$ after major abdominal surgeries with a related mortality of $10 \%$ e $45 \%$ [5]. Despite advances in operative techniques and risk control methods during recent years, the incidence of WD remains high [6,7]. Surgeon expertise, type of incision, suturing material, surgical site infection, nutritionalstatus, persistent cough, abdominal distension, leakage ofpancreatic enzyme, anemia, obesity, diabetes, jaundice, oldage, emergent operation, particular procedures such as colonsurgery, and late wound healing due to malignancy have allbeen suggested to predispose patients toabdominal wound dehiscence.Some of these factors are unavoidable [5,9,10,11]. The retention sutures are one of the recommendedtechniques for reducing disruption of fascia in vulnerablereoperated cases. However, considering the associated painand morbidity, no benefits have been observed that justify theuse of retention sutures as a routine method [12-17].

AIM OF THE STUDY: To evaluate the effect of prophylactic retention sutures in patients with a high risk for wound dehiscence who underwent midline laparotomy. 
PATIENTS AND METHODS: This prospective study was carried out in Al-shatra general hospital and Al-hussain teaching Hospital, Department of Surgery between December 2011 to November 2013. Patients selection:- $\quad$ This prospective randomized controlled double-blinded clinical study by using sealed envelopes to be with or without prophylactic tention sutures ,by a coordinator who was not involved in the research process. The list was concealed from investigators throughout the study.,all patients gave informed consent. During the period of study,patients included who undergoing midline laparotomy, 10-cm surgical incision minimum, and having at least one of the following preoperative risks factors for abdominal dehiscence poor nutritional status (clinical cachexia or hypoalbuminemia); emergent surgery; intra-abdominal infection; malignancy; use of corticosteroids in the last year; uremia; hemodynamic instability (BP <90mmHg); hemoglobin $<10 \mathrm{mg} / \mathrm{dL}$; abdominal distension (due to ascites or prolonged ileus); chronic pulmonary diseases; clinical jaundice (total bilirubin $>3 \mathrm{mg} / \mathrm{dL}$ ); diabetes mellitus; and age $>60$ years[10,18-27]. Patients younger than 12 years and those with an incision length of $<10 \mathrm{~cm}$ were excluded from the study. One hundred fifty(150) cases were randomized to form two groups with 75 patients each: a prophylactic group by using retention sutures and an non prophylactic group. A central randomization for both hospitals was performed.

Two patients in the non prophylactic group and three patients in the prophylactic group died within 2 wks of surgery due to causes unrelated to abdominal dehiscence and were excluded from the study. Therefore 145 patients were evaluated (there were 72 cases with prophylactic qroup, and 73 cases without prophylactic sutures, control group). Indications of surgery were categorized as GIT malignancy, Intestinal obstruction, GIT bleeding, intra-abdominal sepsis, trauma, and miscellaneous.

Table (1) General characteristics of the patient groups with and without prophylactic retention sutures

\begin{tabular}{||l|c|c|l||}
\hline \hline Characteristic & prophylactic group & Nonprophylactic group & $P$ VALUE \\
\hline Sex f/m & $20 / 52$ & $19 / 54$ & 0.766 \\
\hline Age(years) & $13-80$ & $13-70$ & 0.682 \\
\hline Number of risk factors & $2-1.5$ & $2.4-1.2$ & 0.822 \\
\hline Length of incision(cm) & $20+6.3$ & $20+5.6$ & 0.306 \\
\hline Duration of operation (min) & $60-140$ & $60-140$ & 0.119 \\
\hline Indications of laparotomy: & $10(13.5 \%)$ & $8(10.9 \%)$ & 0.237 \\
\hline Intestinal obstruction & $31(42.6 \%)$ & $29(39.5 \%)$ & 0.306 \\
\hline Trauma & $15(20.3 \%)$ & $17(23.1 \%)$ & 0.814 \\
\hline Intraabdominal infection & $7(9.8 \%)$ & $5(6.8 \%)$ & 0.245 \\
\hline Malignancy & $3(4.1 \%)$ & $4(5.4 \%)$ & 0.326 \\
\hline Gastrointestinal bleeding & $34 / 50$ & $37 / 49$ & 0.163 \\
\hline Miscellaneous &
\end{tabular}

The $\mathrm{p}$ value is $>0.05$, the differences between two groups are insignificant.

\section{METHODS:}

All patients underwent general anesthesia, following intubation the patient was prepared and draped in the standard fashion. In the non prophylactic(control)groupStandard midline incision and continuous mass closure technique was used in each case using a running looped $1 / 0$ nylon string located $1 \mathrm{~cm}$ from the edge of the linea alba with $1-\mathrm{cm}$ intervals. Subcutaneous tissue was sutured by interrupted sutures of 3/0 vicryl and skin was closed using interrupted suture of 3/0 nylon. In the prophylactic group, the fascia was sutured using the same technique as the non prophylactic group; however, retention sutures were added using a 1/0 nylon string every $10 \mathrm{~cm}$ and contained $5 \mathrm{~cm}$ of the skin, subcutaneous tissue, rectus muscle, and abdominal fascia (exceptperitoneum) on each side. All fascia closures were performed by tow attending surgeons who adhered strictly to the protocol. Occurrence of abdominal dehiscence was assessed daily by precise examination of the wound. When wound disruption and/or secretions were observed, digital examination of wound depth was performed to evaluate the integrity of the fascia. When the clinical findings were not conclusive, we performed ultrasonography to assessthe fascia. Other postoperative outcomes included evisceration, need to reoperatedue to abdominal wound dehiscence, wound infection (based on clinical findings), postoperative pain, length of postoperative hospital stay, and post-dehiscence in-hospital mortality. Postoperative pain was measured using the visual analog scale (VAS, 0-10 scale) by nurses masked to the patient group. Retention sutures were removed 3 or 4 wks postoperatively when they were loose. The statistical analysis was performed by the statistical software SPSS(version 14 for Windows) using the $\mathrm{x} 2$ test. For the comparison of variables the MannWhitney $\mathrm{U}$ test was applied to compare the variables between the prophylactic and non prophylactic subjects .The results were considered significant at $\mathrm{P}<0.05$. 


\section{RESULTS}

One hundred fifty patients were subjected to midline laparotomy due different causes included in our study, with 75 patients in each group. Two patients in thenonprophylactic group group and three patients in the prophylactic groupdied within two weeks of surgery due to causes unrelated to abdominal wound dehiscence and were excluded from our study.so 72 patients underwent prophylactic retention sutures and 73 patients considered as control non prophylactic group. General characteristics of the patient groups 1, typesof surgeries, length of surgical incision, number of riskfactors and durations of operations are summarized in Table 1. As Table 1 shows, no statistically significant differences were observed between the two groups of patients $(\mathrm{P}>0.05)$. preoperative risk factors for abdominal wound dehiscence in each not show any statistical differences (Table 2,Figure 1) The incidence of abdominal wound dehiscence was 3 patients (4\%) in the prophylactic group and 10 patients $(13.3 \%)$ in the control (nonprophylactic )group $(\mathrm{P}=0.007)$ therefore it is significant. Abdominal evisceration occurred in 1 patient $(0.7 \%)$ in the prophylactic group compared to 2 patients $(2.7 \%)$ in the nonprophylactic group $(\mathrm{P}=0.51)$. Wound infection occurred in $12(15.8 \%)$ and 10 patients $(13.8 \%)$ in the prophylactic and nonprophylacticgroups, respectively $(\mathrm{P}=0.371)$, all these result considered as non significant statistically(Table 3,Figure 2). The pain scores were not significantly different between two groups $(\mathrm{P}>0.05)$, for the prophylactic group,postoperative pain, measured on VAS, was $7.4_{-} 1.6$ on thefirst day, $6.2_{-} 1.8$ on the second day, $4.5_{-} 1.7$ on the third day while, for the nonprophylactic patients, postoperative pain was 7.3 _ 2.3 on the first day, $5.8 \_2.0$ on the second day, 4.1 _ 1.2 on the third day(Table 3,Figure 2) Reoperation after abdominal wound dehiscence was performed tomanage wound dehiscence in all these patientsexcept for one patient in the prophylactic group that was managedconservatively. The fascia of this patient was partially disruptedin the epigastrium, but only the preperitoneal fat wasexposed; therefore, it was managed by frequent change of dressing andthrough secondary healing. Total hospital mortality, postdehiscence deaths and postoperativehospital stay didnot show statistically significant differences (Table 3,Figure 2). Onepost-dehiscence death in the prophylactic group was due to sepsis. Three post-dehiscence mortalities in the nonprophylactic group were due to myocardial infarction, heart failure and renal failure, and pulmonary embolisim(Table 3, Figure 2).

Table (2). Preoperative risk factors of prophylactic and "non- prophylactic" group.

\begin{tabular}{|c|c|c|c|c|c|}
\hline \multicolumn{6}{|c|}{ Distribution of Preoperative Risk factors of abdominal dehiscence } \\
\hline Risk factors & Nonprophylactic group & $\%$ & prophylactic group & $\%$ & $P$ value \\
\hline Age $>60$ years & $30 / 72$ & $44.8 \%$ & $32 / 73$ & $45.8 \%$ & 0.907 \\
\hline jaundice & $10 / 72$ & $14.3 \%$ & $13 / 73$ & $17.3 \%$ & 0.525 \\
\hline Diabetis & $12 / 72$ & $15.6 \%$ & $8 / 73$ & $10.8 \%$ & 0.234 \\
\hline uremia & $2 / 72$ & $2.7 \%$ & $4 / 73$ & $4.7 \%$ & 0.684 \\
\hline$H b<10 \mathrm{~g} / \mathrm{dl}$ & $36 / 72$ & $50.2 \%$ & $35 / 73$ & $49.3 \%$ & 0.541 \\
\hline Emergency laparotomy & $29 / 72$ & $39.3 \%$ & $32 / 73$ & $43.8 \%$ & 0.479 \\
\hline Maliganacy & $40 / 72$ & $56.3 \%$ & $35 / 73$ & $49.3 \%$ & 0.484 \\
\hline Corticosteroid use & $2 / 72$ & $2.6 \%$ & $4 / 73$ & $5.4 \%$ & 0.378 \\
\hline Abdominal distention & $13 / 72$ & $17.7 \%$ & $14 / 73$ & $19.5 \%$ & 0.763 \\
\hline Intraabdominal infection & $19 / 72$ & $25.9 \%$ & $21 / 73$ & $27.7 \%$ & 0.795 \\
\hline $\begin{array}{l}\text { Unstable hemodynamic } \\
\text { state }\end{array}$ & $5 / 72$ & $5.9 \%$ & $6 / 73$ & $7.7 \%$ & 0.636 \\
\hline
\end{tabular}


The $\mathrm{p}$ value is $>0.05$, the differences between two groups are insignificant.

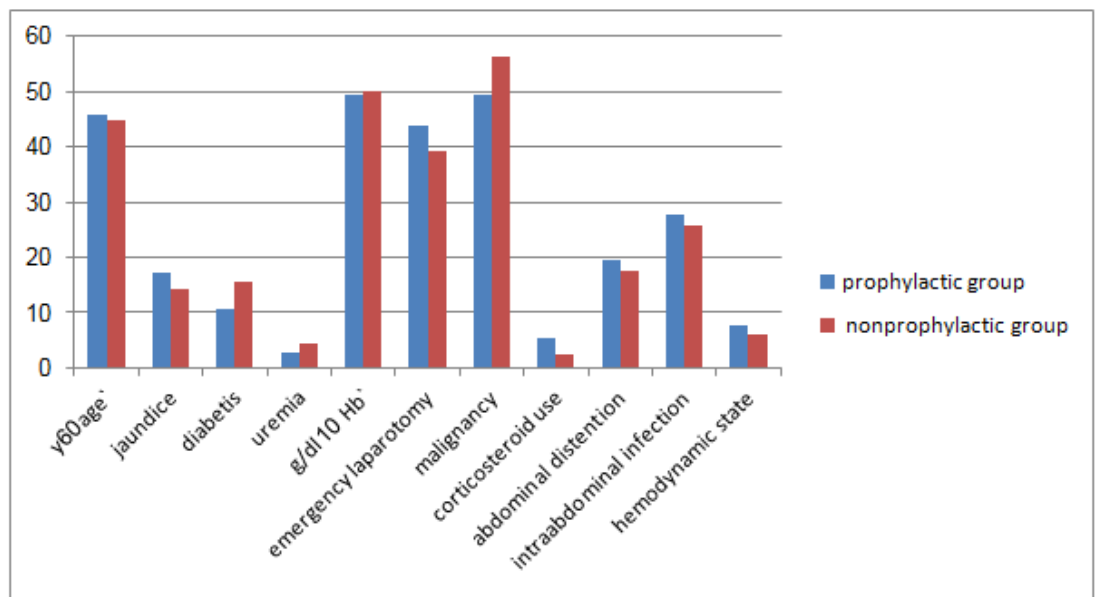

Fig (1).show incidence of risk factors in both group

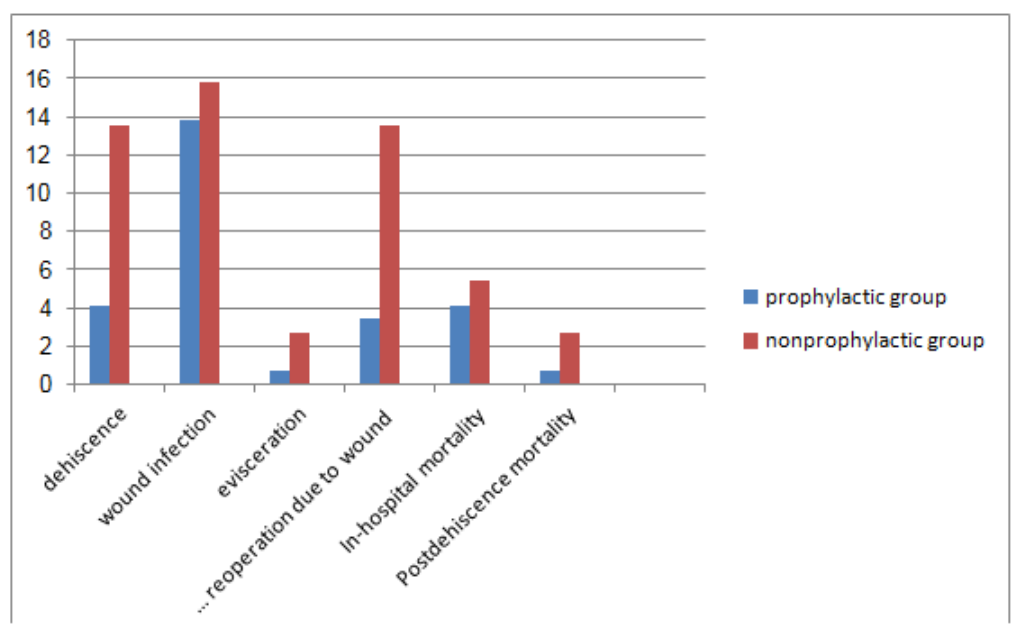

Fig (2).show incidence of postoperative outcome of both group

Table (3).Postoperative fellow up and complications in each group

\begin{tabular}{|c|c|c|c|c|c|}
\hline Outcome & prophylactic group & $\%$ & Nonprophyactic group & $\%$ & $P$ value \\
\hline Dehiscence & $3 / 72$ & $4.1 \%$ & $10 / 73$ & $13.5 \%$ & 0.007 \\
\hline Wound infection & $10 / 72$ & $13.8 \%$ & $12 / 73$ & $15.8 \%$ & 0.371 \\
\hline Evisceration & $1 / 72$ & $0.7 \%$ & $2 / 73$ & $2.7 \%$ & 0.51 \\
\hline VAS $1^{\text {st }}$ day & $7.3 \_2.3$ & & $7.4 \_1.6$ & & 0.939 \\
\hline$V A S 2^{\text {nd }}$ day & $5.8_{-} 2$ & & $6.2 \_1.8$ & & 0.090 \\
\hline$V A S 3^{\text {rd }}$ day & $4.1_{-} 1.2$ & & $4.5_{-} 1.7$ & & 0.078 \\
\hline $\begin{array}{l}\text { Postoperative hospital } \\
\text { stay }(d)\end{array}$ & $21.3_{-} 6.9$ & & $20.4_{-} 5.6$ & & 0.332 \\
\hline $\begin{array}{l}\text { Reoperation due to } \\
\text { wound dehiscence }\end{array}$ & $2 / 72$ & $3.4 \%$ & $10 / 73$ & $13.5 \%$ & 0.003 \\
\hline In-hospital mortality & $3 / 72$ & $4.1 \%$ & $4 / 73$ & $5.4 \%$ & 0.785 \\
\hline $\begin{array}{l}\text { Postdehiscence } \\
\text { mortality }\end{array}$ & $1 / 72$ & $0.7 \%$ & $2 / 73$ & $2.7 \%$ & 0.622 \\
\hline
\end{tabular}




\section{DISCUSSION:-}

The discussion on the subject of abdominal wound dehiscence is as old as the history of modern operative sur- gery. The peri-operative mortality and long term morbidity associated with the condition need medical surgical pre- ventive measures to be taken. Abdominal wound dehiscence is a devastating incident that can cause pain, mentaldistress, infectious complications, and financial burdens forthe patient, as well as complications including eviscerationand reoperation $[6,8,9,13,28]$. Surgeon expertise, type of incision, suturing material, surgical site infection, nutritional status, persistent cough, abdominal distension, leakage of pancreatic enzyme, anemia, obesity, diabetes, jaundice, old age, emergent operation, particular procedures such as colon surgery, and late wound healing due to malignancy have all been suggested to predispose patients to abdominal wound dehiscence.Some of these factors are unavoidable [5,13,10,29,30]. Different surgical techniques for closing the wound should be carefully considered [31]. Suture materials are of great importance in providing sufficient strength and influencing adverse events [7].

Some authors have proposed the application of thick or retention sutures as a preventive strategy to eliminate or reduce the occurrence of wound dehiscence[25,30,32-34] Retention sutures have already been shown to reduce the rate of WD after surgery $[6,14,15,30]$, and their use has also been suggested as a treatment choice for managing fascial dehiscence $[5,35]$. however, due to the subsequent pain, postoperative discomfort, and skinmaceration, routine application of this technique has not been well accepted. Considering the controversies involved in using this method for the prevention of abdominalwound dehiscence, our study included only patients at a high risk for developing abdominalwound dehiscence who would benefit the most from prophylactic retention sutures. Complications such as intestinal damage [13,16,36], skin maceration and cutting lesions [37,38,16,36], surgical site infections, and patient pain ordiscomfort $[6,27]$ prohibit the surgeons from performing this technique. However, in the presence of a high possibility for developing abdominalwound dehiscence due to the accompanying conditions, thebenefits of retention sutures may outweigh the disadvantagesand the technique should be considered. However, in the presence of a high possibility for developing abdominal wound dehiscence due to the accompanying conditions, thebenefits of retention sutures may outweigh the disadvantagesand the technique should be considered. abdominal wound dehiscence can be prevented by certain strategies, such as using a vacuum assisted closure in patient with compromised healing or using tension free mesh techniques in order to reduce the tension of the abdominal wall[39 ].

ZhamakKhorgami et al., in a study with a large sample size, reporteda lower rate of incidence for abdominal wound dehiscence when retention sutures are used at the time of wound closure. They suggested that the selection of patients from the high-risk population is essential for raising the benefits against the costs of preventive approaches[40]. In contrast, Hubbard andRever concluded there were no advantages in applying retention sutures for the prevention of abdominal wound dehiscence [41]. Our study showed a lower incidence of abdominal wound dehiscence in prophylactic group in which just 3 from 72 cases develop wound dehiscence in contrast to nonprophylactic group in which 10 from 73 cases develop dehiscence which significant. The cases for this study were selectedfrom high-risk patients (two or more risk factors) and the findings would suggestthat this method, as a preventive strategy, benefits sucha population. The decreased incidence of abdominal wound dehiscence in our study is inline with some other studies. Goligher et al.,suggested that reinforcing the routine methods of closure with retention sutures or application of a wire suture would result in fewer cases of dehiscence. However, we should note that the incidence of dehiscence in our study $(8.8 \%)$ was higher compared to others due to enrolling high-risk patients [42]. Evisceration,post operative wound infection ,mortality rate and post operative pain were less frequent in the prophylactic group, but this finding lacks statistical significance and is not conclusive given the small number of events. Our findings suggest, patient selection among the high risk population with multiple risk factors for abdominal wound dehiscence is a prudent approach to apply retention sutures as a prophylactic routinefor prevention of abdominal wound dehiscence. With such a treatment approach, the risks of developing dehiscence would outweigh the complications. limitation of our study was the small sample of cases of laparotomies in patients with risk factors of abdominal wound dehiscence. Furthermore, the short follow-up period for observingthe development of incisional hernia should be replaced by a much longer period to assess development of incisional hernia.

\section{CONCLUSION}

Our conclsion that prophylactic retention sutures can decrease the incidence of abdominal wound dehiscence but although there is decrease incidence of post operative evisceration ,wound infection and post operative pain , there was no significant difference. 


\section{REFERENCES}

[1] Chin G, Diegelman R, Schultz G: Cellular and molecular regulationof wound healing. In Wound healing Edited by: Falabella A, Kirschner R. Boca Roton FL; Taylor, Francis Group; 2005:17-37. Hugh TB: Abdominal wound dehiscence, editorial comment. Aust NZ J Surgery 1990, 60:153-155

[2] Holmes J.D. Classification of wounds and their mana gement. In: Lumley JSP, Caraven JL. SurgInt 1999;45:63-5 A. G. Greenberg and R. P. Saik, "Wound Dehiscence - Pathophysiology and Prevention," Archives of Surgery, Vol. 114, No. 2, 1979, pp. 143-146.

[3] Gislason H, Gronbech JE, Soreide O. Burst abdomen and incisional hernia after major gastrointestinal operationsdComparison of three closure techniques. Eur J Surg 1995;161:349.

[4] Penninckx FM, Poelmans SV, Kerremans RP, et al. Abdominal wound dehiscence in gastroenterological surgery. Ann Surg $1979 ; 189: 345$.

[5] Wissing J, van Vroonhoven TJ, Schattenkerk ME, et al. Fascia closure after midline laparotomy: Results of a randomized trial. Br J Surg 1987;74:738.

[6] Riou JP, Cohen JR, Johnson H Jr. Factors influencing wound dehiscence. Am J Surg 1992;163:324.

[7] Armstrong CP, Dixon JM, Duffy SW, et al. Wound healing in obstructive jaundice. Br J Surg 1984;71:267. 10.Col C, Soran A, Col M. Can postoperative abdominal wound dehiscence be predicted? Tokai J ExpClin Med 1998;23:123.

[8] Sørensen LT, Hemmingsen U, Kallehave F, et al. Risk factors for tissue and wound complications in gastrointestinal surgery. Ann Surg 2005;241:654.

[9] Gislason H, Gronbech JE, Soreide O. Burst abdomen and incisional hernia after major gastrointestinal operationsdComparison of three closure techniques. Eur J Surg 1995;161:349.

[10] Adams G, Richter RM, Levowitz BS. A safe method of closure with retention sutures. SurgGynecolObstet 1973;136:981.

[11] Banerjee SR, Daoud I, Russell JC, et al. Abdominal wound evisceration. CurrSurg 1983;40:432. 15. Miles RM, Moore M, Fitzgerald D, et al. The etiology and prevention of abdominal wound disruption: An analysis of 177 cases. Am Surg 1964;30:566.

[12] Ponce LC, Morgan MW. A safer wire retention suture. Am Surg 1970;36:509.

[13] Stivala OG. Retention suture plates. SurgGynecolObstet 1983;157:77.

[14] Bucknall TE, Cox PJ, Ellis H. Burst abdomen and incisional hernia: A prospective study of 1129 major laparotomies. Br Med J (Clin Res Ed) 1982;284:931.

[15] Poole GV Jr. Mechanical factors in abdominal wound closure: The prevention of fascial dehiscence. Surgery 1985;97:631.

[16] Riou JP, Cohen JR, Johnson H Jr. Factors influencing wound dehiscence. Am J Surg 1992;163:324.

[17] Ma kela“ JT, Kiviniemi H, Juvonen T, et al. Factors influencing wound dehiscence after midline laparotomy. Am J Surg 1995; 170:387.

[18] Webster C, Neumayer L, Smout R, et al. Prognostic models of abdominal wound dehiscence after laparotomy. J Surg Res 2003;109:130.

[19] Khan M, Naqvi AH, Irshad K, et al. Frequency and risk factor of abdominal wound dehiscence. J Coll Physicians Surg Pak 2004;14:355.

[20] Afzal S, Bashir MM. Determinants of wound dehiscence in abdominal surgery in public sector hospital. Annals of King Edward Medical University 2008;14:110. 25. Waldhausen JH, Davies L. Pediatric postoperative abdominal wound dehiscence: Transverse versus vertical incisions. J Am CollSurg 2000;190:688.

[21] Van Ramshorst GH, Nieuwenhuizen J, Hop WCJ, et al. Abdominal wound dehiscence in adults: Development and validation of a risk model. World J Surg 2010;34:20.

[22] Rink AD, Goldschmidt D, Dietrich J, et al. Negative side-effects of retention sutures for abdominal wound closure. A prospective randomised study. Eur J Surg 2000; 166:932.

[23] Wahl W, Menke H, Schnutgen M, et al. [Fascia dehiscencedCause and prognosis]. Chirurg 1992;63:666.

[24] Sørensen LT, Hemmingsen U, Kallehave F, et al. Risk factors for tissue and wound complications in gastrointestinal surgery. Ann Surg 2005;241:654.

[25] Reitamo J, Moller C. Abdominal wound dehiscence. ActaChir Scand 1972;138:170.

[26] Richards PC, Balch CM, Aldrete JS. Abdominal wound closure. A randomized prospective study of 571 patients comparing continuous vs. interrupted suture techniques. Ann Surg 1983;197:238.

[27] Doughty DB. Preventing and managing surgical wound dehiscence. Adv Skin Wound Care 2005;18:319.

[28] Waldrop J, Doughty D. Wound healing physiology. In: Bryant R, editor. Acute and chronic wounds: Nursing management. 2nd ed. St. Louis, MO: Mosby; 2000. p. 17.

[29] Posthauer M, Thomas D. Wound care essentials. In: Baranoski S, Ayello E, editors. Nutrition and wound care. Philadelphia: Lippincott: Williams and Wilkins; 2004. p. 379. 35. Carlson MA. Acute wound failure. SurgClin North Am 1997; 77:607.

[30] Chavez-Cartaya R, Jiron-Vargas A, Pinto S, et al. Adjustable nylon ties for abdominal wall closure. Am J Surg 1992; 163:609.

[31] Boissel P, Jamart J, Grumillier P, et al. A new technique for closing abdominal incisions in patients with poor wound healing. Am J Surg 1982;143:380.

[32] Matsuoka J, Gohchi A, Kamikawa Y, et al. Chopstick retention suture for the closure of abdominal wounds. J Am CollSurg 1995;181:471..

[33] Heller L, Levin S, Butler C: Management of abdominal wound dehiscence using vacuum assisted closure in patients with compromised healing. Am J Surg 2006, 191:165-172

[34] ZhamakKhorgamietal: Prophylactic retention sutures in midline laparotomy in high-risk patients for wound dehiscence.journal homepage: www.JournalofSurgicalResearch.com.

[35] Hubbard TB Jr, Rever WB Jr. Retention sutures in the closure of abdominal incisions. Am J Surg 1972;124:378.

[36] Goligher JC, Irvin TT, Johnston D, et al. A controlled clinical trial of three methods of closure of laparotomy wounds. Br J Surg 1975;62:823. 